\title{
Morphology Observation of Novel 3, 4-Bis-Alkyloxycarbonyl-Hexanedioic Acid Monolayer on Water Surface by Dropping Method
}

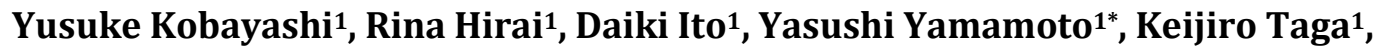 \\ Zameer Shervani ${ }^{*}$ \\ ${ }^{1}$ Department of Life Science and Material Engineering, Nagoya Institute of Technology, Nagoya, Japan \\ ${ }^{2}$ Tohoku Foods Corporation Limited, Sendai, Japan \\ Email: “yamamoto.yasushi@nitech.ac.jp, "shervani.nanotech@gmail.com
}

Received 22 March 2016; accepted 14 May 2016; published 17 May 2016

Copyright (C) 2016 by authors and Scientific Research Publishing Inc.

This work is licensed under the Creative Commons Attribution International License (CC BY).

http://creativecommons.org/licenses/by/4.0/

(c) (i) Open Access

\section{Abstract}

We have investigated the morphology of novel 3, 4-bis-alkyloxycarbonyl-hexanedioic acid monolayer (alkyl chain number $n=12,14$, and 16) formed on the water surface by dropping method using Surface Tension measurement (STm) and Brewster Angle Microscopy (BAM). Surfactant of 3, 4-bis-alkyloxycarbonyl-hexanedioic acid has a gemini type structure. $\pi$ - $A$ isotherm curves (STm) of two gemini surfactants of $n=12$ and 14 showed gradual increase in surface pressure, while that of $n=16$ showed gradual increase, a stagnant range and finally steep increase. BAM observation showed each image corresponding to the result of STm measurement. From STm and BAM results of surfactants, it is found that surfactant of $n=12$ and 14 form Liquid-Expand (LE) type monolayer, while that of $n=16$ formed Liquid-Condensed (LC) type monolayer including LE-LC phase transition.

\section{Keywords}

3, 4-Bis-Alkyloxycarbonyl-Hexanedioic Acid, Gemini Surfactants, Gemsurfs, Monolayer, Morphology

\section{Introduction}

Gemini surfactant essentially has a dimer structure at which two monosurfactants are connected by a spacer

*Corresponding authors.

How to cite this paper: Kobayashi, Y., Hirai, R., Ito, D., Yamamoto, Y., Taga, K. and Shervani, Z. (2016) Morphology Observation of Novel 3, 4-Bis-Alkyloxycarbonyl-Hexanedioic Acid Monolayer on Water Surface by Dropping Method. Journal of Biophysical Chemistry, 7, 43-48. http://dx.doi.org/10.4236/jbpc.2016.72004 
group near those hydrophilic head groups [1]-[3]. Compared to the corresponding monosurfactant, gemini surfactant has various characteristics such as low Krafft temperature, low critical micelle concentration, and a low surface tension. Since Zana and Talmon [1] synthesized gemini surfactant and studied their solution properties, these surfactant are being used in various biological and industrial applications such as vehicles for gene delivery into cells [4], understanding cell fusion and fission processes [5], effective gelation of water [6], and soft template for anisotropic gold particles [7]. Studies related to air-water interface such as aggregation behavior of cationic gemini surfactants and interaction with nucleoside bases [8], formation of gemini surfactant-DNA complex monolayer for surface pattering and nanofabrication [9], miscibility between gemini surfactant-stearic acid for elucidation of biological membrane and self-assembly [10] have been also reported.

In year 2004, Chukyo Yushi Co., Japan [11] synthesized a novel gemini surfactant 3, 4-bis-alkyloxycarbonylhexanedioic acid commonly known as "Gemsurf" whose structure is shown in Figure 1. Gemsurf is different from previously reported gemini surfactant in following characteristics: two hydrophilic head groups are connected directly by one carbon-carbon single bond. Gemsurf is also similar to the structure of amphiphilic molecule of biomembranes. Gemsurf commercial synthesis has been established and surfactant currently is being used in cosmetics and emulsion preparations, as dispersants, bubble stabilizers, and cleaners. Solution properties of Gemsurf-inorganic and -organic substances [11] and complex of Gemsurf-monosurfactants [12] have been investigated. However, there is no report of surface properties of Gemsurf behavior at air-water interface.

In this context we have investigated for the first time the morphology of the novel Gemsurf monolayer formed on the water surface by the dropping method [13] using Surface Tension measurement (STm) and Brewster Angle Microscopy (BAM).

\section{Experimental}

\subsection{Materials}

Gemsurf of alkyl chain numbers $n=12,14$, and 16 in Figure 1 was gifted from Chukyo Yushi Co., Japan and used without further purification. Spreading solvent chloroform (99.0\%) was procured from Wako Pure Chemical Industries Ltd., Osaka, Japan. Purified water with conductance $<0.07 \mu \mathrm{S} / \mathrm{cm}$ for subphase was obtained using a Super Water Purifying System (WL-21P; Yamato Scientific Corp. Ltd., Tokyo, Japan).

\subsection{Monolayer Formation}

Details of the monolayer formation using the dropping method have been reported in a previous article [13]. 1 $\mathrm{mM}$ solution of Gemsurf-chloroform was spread on the purified water surface using a $100 \mu \mathrm{l}$ microsyringe (Ge-0583-04; Hamilton Co., Nevada, USA). Spreading was conducted at $1 \mu \mathrm{l} / \mathrm{min}$ intervals. Gemsurf-chloroform droplet was dropped gently and the next drop was added after the previous droplet expanded on the water surface. The completion of monolayer formation was confirmed by the fact that the formation of lenses of droplet was observed on the water surface, and value of surface tension as described in Sections 2.3 did not change even if droplet continued being dropped.

\subsection{Surface Tension Measurement (STm)}

STm for each Gemsurf monolayer was carried out on a Surface Tensiometer (CBVP-A3; Kyowa Interface Science Corp. Ltd.). As mentioned in Section 2.2, Gemsurf-chloroform solution was spread on the purified water surface by dropping method with a platinum Wilhelmy plate. The surface pressure of each monolayer was

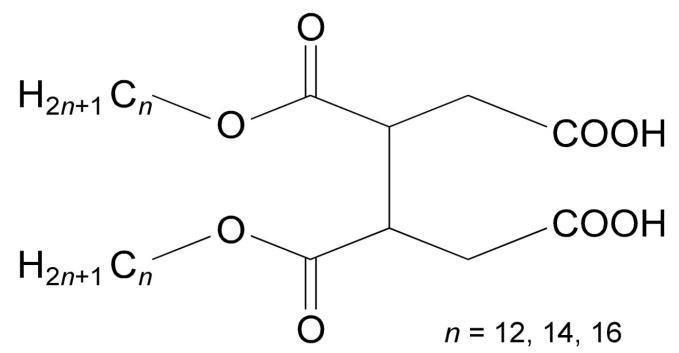

Figure 1. Molecular structure of Gemsurf $(n=12,14$, and 16). 
recorded as a function of spreading volume at $26.0^{\circ} \mathrm{C} \pm 0.2^{\circ} \mathrm{C}$ and measurements were conducted more than three times and experimental errors were $\pm 0.1 \mathrm{mN} / \mathrm{m}$.

\subsection{Brewster Angle Microscopy (BAM)}

Brewster angle microscopy (BAM) for visualizing the morphology of each Gemsurf monolayer was carried out on a Brewster angle microscope (EMM633K; Filgen Inc., Nagoya, Japan) and USB-CAP type (SD-USB2CUP3; AREA Co. Ltd, Tokyo, Japan) imaging analysis software. A p-polarized light of $632.8 \mathrm{~nm}$ wavelength was irradiated at the Brewster angle of $53.1^{\circ}$ on each monolayer surface. The reflected light was detected by a CCD camera (C5948-70; Hamamatsu Photonics, Hamamatsu, Japan). The lateral resolution of BAM was about $1 \mu \mathrm{m}$. The formation process of each monolayer was observed at the arbitrary surface pressure as described in Section 3.2. The BAM measurement was conducted at $26^{\circ} \mathrm{C} \pm 1^{\circ} \mathrm{C}$ and measurements were conducted more than three times.

\section{Results}

\section{1. $\pi$-A Isotherm Curves}

Figure 2 shows the surface pressure versus molecular area $(\pi-A)$ isotherm curves of three Gemsurf surfactants ( $n=12,14$, and 16) monolayers obtained at $26^{\circ} \mathrm{C}$ using the dropping method. Horizontal axis represents molecular area calculated from molecular numbers in the dropping volume. Vertical axis represents surface pressure measured when enough time passed after dropping of Gemsurf-chloroform droplet and the pressure value became constant. At Gemsurf of $n=12$ and 14, surface pressure values increased 10 and 5 units each on surface pressure scale, respectively.

Gemsurf of $n=12$ has presented a $\pi$-A isotherm curve similar to a Liquid-Expand (LE) type monolayer [14]. Therefore, the surface pressure increases gradually from about $1.2 \mathrm{~nm}^{2}$ molecular area and the pressure increased monotonously up to $40 \mathrm{mN} / \mathrm{m}$. The shape of the curve is smooth and different from the steep curve observed in a liquid-condensed (LC) type monolayer. The limiting molecular area $\left(A_{0}\right)$ is calculated to be $c a .0 .74 \pm$ $0.02 \mathrm{~nm}^{2} /$ molecule.

Gemsurf of $n=14$ has also presented an isotherm curve similar to LE type monolayer, the surface pressure increases gradually from about $1.1 \mathrm{~nm}^{2}$ molecular area and the pressure increased monotonously up to $39 \mathrm{mN} / \mathrm{m}$. The shape of curve is smooth and $A_{0}$ is calculated to be $c a .0 .69 \pm 0.02 \mathrm{~nm}^{2} /$ molecule.

Gemsurf of $n=16$, on the other hand, has presented a different isotherm curve compared to that of $n=12$ and 14. Therefore, the surface pressure increased gradually at $1.1 \mathrm{~nm}^{2}$ molecular area and after a stagnation of

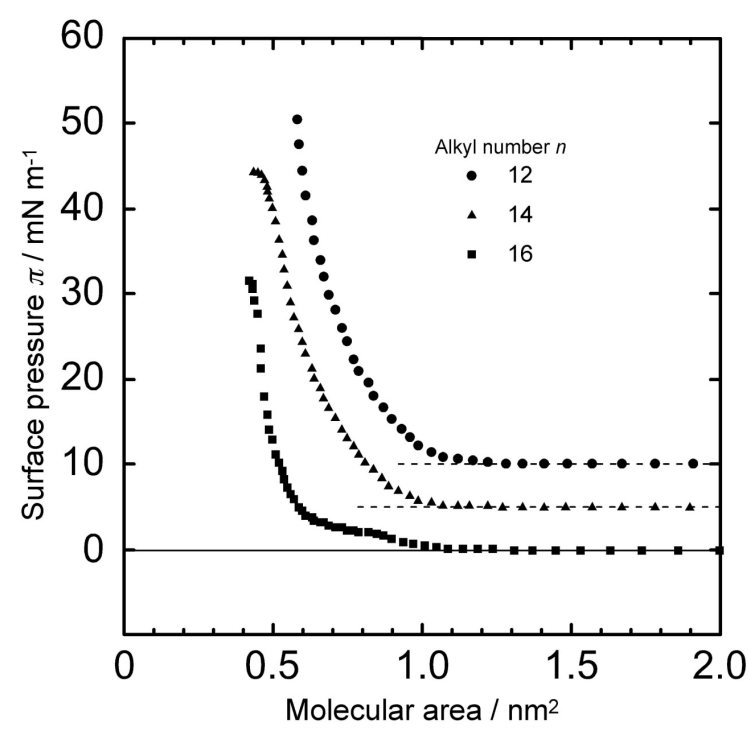

Figure 2. Surface pressure versus molecular area $(\pi-A)$ isotherm curve of Gemsurfs $(n=12,14$, and 16$)$ at $26^{\circ} \mathrm{C}$. $\bullet:$ Gemsurf of $n=12, \boldsymbol{\Delta}: n=14, \mathbf{m}: n=16$. 
surface pressure at $2 \mathrm{mN} / \mathrm{m}$ in molecular area range of 0.70 to $0.85 \mathrm{~nm}^{2}$, it increased steeply up to $32 \mathrm{mN} / \mathrm{m}$. $A_{0}$ is calculated to be $c a$. $0.53 \pm 0.02 \mathrm{~nm}^{2} /$ molecule. Such curve is similar to that of dipalmitoyl phosphatidyl choline (DPPC) monolayer [13]-[15] at which the surface pressure increases gradually at low surface pressures as a LE state and it also shows a steep increase at high surface pressures as a LC state. The LE-LC phase transition occurs at a surface pressure $10 \mathrm{mN} / \mathrm{m}$.

\subsection{BAM Observation}

Figure 3 shows a series of BAM images on three Gemsurf $\left(n=12,14\right.$, and 16) monolayers obtained at $26^{\circ} \mathrm{C}$ using the dropping method. BAM observations were performed on each monolayer at the dropping volume corresponding to characteristic of four surface pressures of each monolayer.

For Gemsurf of $n=12$ (Figures 3(a)-(d)), we obtained similar images like that observed in the case of LE type monolayer. Therefore, at a low surface pressure of $1 \mathrm{mN} / \mathrm{m}((\mathrm{a}))$, the image of monolayer was a little brighter compared to that of water surface. According to an increase in surface pressure (6 and $18 \mathrm{mN} / \mathrm{m}$, (b) and (c)), those images became brighter and the formation of monolayer continued homogeneously. At $35 \mathrm{mN} / \mathrm{m}$ ((d)), the image became further brighter and finally the homogeneous monolayer was formed and completed. Such a gradual increase in brightness of monolayer over the pressure range corresponded to the gradual increase in the surface pressure on $\pi$ - $A$ isotherm curve (Figure 2).

For Gemsurf of $n=14$ (Figures 3(e)-(h)), images were similar to LE type monolayer as observed in $n=12$. According to an increase in surface pressure (1, 9, and $26 \mathrm{mN} / \mathrm{m}$, (e), (f), and (g)), those images became brighter over the whole and the formation of monolayer continued homogeneously. At $39 \mathrm{mN} / \mathrm{m}((\mathrm{h}))$, image became further brighter and the homogeneous monolayer was completed. Brightness of image of (h) was somewhat lighter than that of $35 \mathrm{mN} / \mathrm{m}$ of Gemsurf of $n=12((\mathrm{~d}))$. The difference is related to monolayer thickness and its
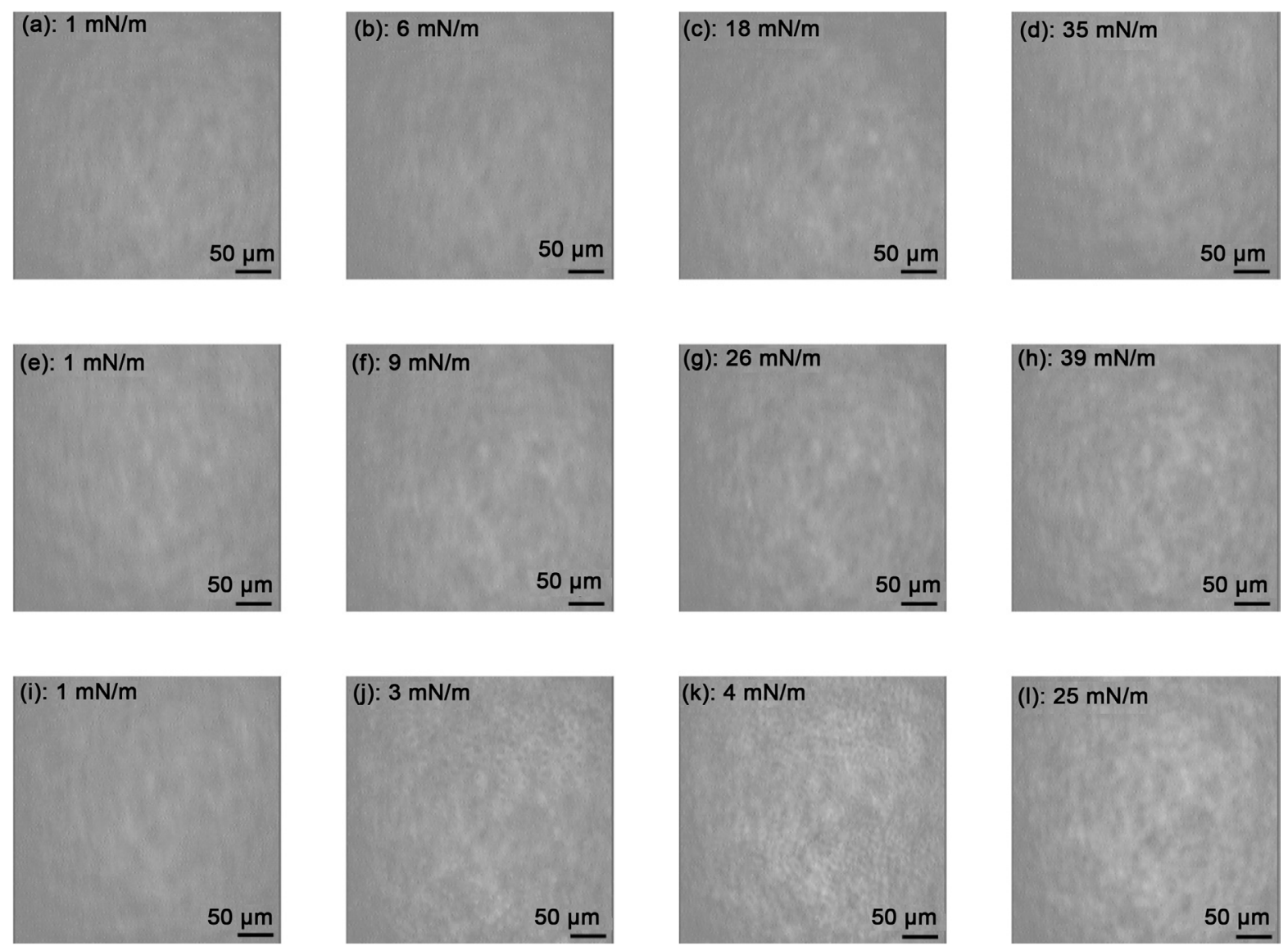

Figure 3. BAM images of Gemsurfs ( $n=12,14$, and 16) on various surface pressures at $26^{\circ} \mathrm{C}$. (a)-(d) Gemsurf of $n=12$, (e)-(h) $n=14$, and (i)-(l) $n=16$. 
refractive index of monolayer [16] [17].

For Gemsurf of $n=16$ (Figures 3(i)-(1)), monolayer images were different from that of Gemsurf of $n=12$ and 14. At a low surface pressure of $1 \mathrm{mN} / \mathrm{m}$ ((i)), image was a little bright. After the LE-LC phase transition started at about $2 \mathrm{mN} / \mathrm{m}$, image ((j)) showed bright dark contrast and hole or island type domain appeared in the image. At a surface pressure of about $4 \mathrm{mN} / \mathrm{m}$ (image $(\mathrm{k})$ ), the contrast became clearer and with assembled domains. At a surface pressure of $25 \mathrm{mN} / \mathrm{m}$ (image (l)) the image became further brighter and homogeneous monolayer was formed on the water surface.

\section{Discussion}

From the results of STm and BAM, it is found that Gemsurf of $n=12$ and 14 is LE type monolayer, while Gemsurf of $n=16$ is LC type monolayer with LE-LC phase transition. In a handbook [14], it has been shown that in LE type monolayer, alkyl chains in monolayer molecules have gauche structure and have random orientation. Whereas, in LC type monolayer, those have trans structure and oriented uniformly on the water surface.

It is known that alkyl chain structure in monolayer molecules maintains the original bulk structure and properties of molecules. Differential Scanning Calorimetry (DSC) has been conducted of Gemsurf of $n=12$, 14, and 16. Gemsurf of $n=12$ showed the peak of phase transition at 0.4 and $92.3{ }^{\circ} \mathrm{C}$. In $n=14$, phase transitions were at $24.8^{\circ} \mathrm{C}$ and $95.0^{\circ} \mathrm{C}$, and $n=16$, at $40.8^{\circ} \mathrm{C}$ and $99.3^{\circ} \mathrm{C}$. It seems that the peak appeared at low temperature for each Gemsurf corresponds to the melting of alkyl chain and reorientation of molecules from crystal to liquid crystal phase, and at high temperature it corresponds to the bulk melting point. The phase of the above 3-dimension bulk state does not always reflect the state of 2-dimension monolayer, but DSC results indicate that the Gemsurf of $n=12$ and 14 is a monolayer of gauche structure of alkyl chain and $n=16$ is a monolayer of trans of alkyl chain at $26^{\circ} \mathrm{C}$.

The apparent molecular area of hydrophilic group (dicarboxylic acid part) of Gemsurf $\left(A_{\mathrm{h}-\mathrm{g}}\right)$ was estimated to be $c a .0 .4 \mathrm{~nm}^{2}$ from roughly calculation by molecular model (molecular model kit (MOL-TALOU Models, basic-A set, Koriyama, Japan)). From the value of above molecular area, two alkyl chains of gauche structure in Gemsurf of $n=12$ and 14 are spread larger than area of $A_{\mathrm{h}-\mathrm{g}}$ on the water surface, thereby $A_{0}$ would become a larger value. $A_{0}$ of $n=12$ was larger than that of $n=14$. Because the alkyl chain length of $n=14$ is longer than that of $n=12$, the stronger interaction between alkyl chains of $n=14$ would make $A_{0}$ of $n=14$ small compared to that of $n=12$. In Gemsurf of $n=16$, two alkyl chains have trans structure that would promote the interactions between alkyl chains. As a result, the spread of alkyl chain on the water surface is restrained compared to that of $n=12$ and 14, and also $A_{0}$ of $n=16$ would become smaller than that of $n=12$ and 14 . Molecular area of trans saturated acyl chains $\left(A_{\mathrm{a}-\mathrm{c}}\right)$ is $c a .0 .2 \mathrm{~nm}^{2}$ [18], $A_{0}$ of $n=16$ is slightly larger than $A_{\mathrm{h}-\mathrm{g}}$ and sum of two $A_{\mathrm{a}-\mathrm{c}}$. This indicates that two alkyl chains open between each other or tilt a little from water surface normal.

The above dependence and behavior of alkyl chain length on the measured Gemsurfs monolayer is quite similar to that of dilauroyl phosphatidyl choline (DLPC, $n=12$ ), dimyristoyl phosphatidyl choline (DMPC, $n=14$ ), and dipalmitoyl phosphatidyl choline (DPPC, $n=16$ ) which are biomembrane model substances [14]. Therefore, DLPC and DMPC form LE type monolayer, whereas DPPC forms LC type monolayer including LE-LC phase transition. The alkyl chain of DLPC and DMPC has gauche structure and DPPC has trans. The order of $A_{0}$ is DLPC > DMPC > DPPC. Measured Gemsurfs are quite different from composition and structures of hydrophilic groups and hydrophilic lipophilic balance (HLB) compared to phospholipids, however, Gemsurfs showed monolayer morphology similar to phospholipids formed on the water surface. This implies that Gemsurfs have a possibility with the surfactant ability similar to that of phospholipids. Nakahara and co-workers [19] [20] have reported the importance of the function and the role as lung surfactants by DPPC and palmitic acid molecules. On the pattern of applications of lipid surfactants, we expect that Gemsurfs would not only be used in cosmetic and industrial products but also have applications in in-vivo components to substitute biomolecules.

\section{Conclusion}

We have investigated the morphology of the novel Gemsurfs $(n=12,14$, and 16) monolayer formed on the water surface at $26^{\circ} \mathrm{C}$ using STm and BAM methods. It was found that $n=12$ and 14 formed LE type monolayer and that of $n=16$ LC type monolayer where LE to LC phase transition existed. Morphology of Gemsurf monolayers was similar to those of phospholipids series of biomolecules. Gemsurfs have capable of surface activity as well as phospholipids and also a possibility of application as biosurfactant. 


\section{References}

[1] Zana, R. and Talmon, Y. (1993) Dependence of Aggregate Morphology on Structure of Dimeric Surfactants. Nature, 362, 228-230. http://dx.doi.org/10.1038/362228a0

[2] Menger, F.M. and Keiper, J.S. (2000) Gemini Surfactants. Angewandte Chemie International Edition, 39, $1906-1920$. http://dx.doi.org/10.1002/1521-3773(20000602)39:11<1906::AID-ANIE1906>3.0.CO;2-Q

[3] In, M. and Zana, R. (2007) Phase Behavior of Gemini Surfactants. Journal of Dispersion Science and Technology, 28, 143-154. http://dx.doi.org/10.1080/01932690600991888

[4] McGregor, C., Perrin, C., Monck, M., Camilleri, P. and Kirby, A.J. (2001) Rational Approaches to the Design of Cationic Gemini Surfactants for Gene Delivery. Journal of the American Chemical Society, 123, 6215-6220. http://dx.doi.org/10.1021/ja005681c

[5] Sommerdijk, N.A.J.M., Hoeks, T.H.L., Synak, M., Feiters, M.C., Nolte, R.J.M. and Zwanenburg, B. (1997) Stereodependent Fusion and Fission of Vesicles: Calcium Binding of Synthetic Gemini Phospholipids Containing Two Phosphate Groups. Journal of the American Chemical Society, 119, 4338-4344. http://dx.doi.org/10.1021/ja962303s

[6] Estroff, L.A. and Hamilton, A.D. (2000) Effective Gelation of Water Using a Series of Bis-Urea Dicarboxylic Acids. Angewandte Chemie International Edition, 39, 3447-3450. http://dx.doi.org/10.1002/1521-3773(20001002)39:19<3447::AID-ANIE3447>3.0.CO;2-X

[7] Esumi, K., Hara, J., Aihara, N., Usui, K. and Torigoe, K. (1998) Preparation of Anisotropic Gold Particles Using a Gemini Surfactant Template. Journal of Colloid and Interface Science, 208, 578-581. http://dx.doi.org/10.1006/jcis.1998.5852

[8] Wang, Y., Desbat, B., Manet, S., Aimé, C., Labrot, T. and Oda, R. (2005) Aggregation Behaviors of Gemini Nucleotide at the Air-Water Interface and in Solutions Induced by Adenine-Uracil Interaction. Journal of Colloid and Interface Science, 283, 555-564. http://dx.doi.org/10.1016/j.jcis.2004.09.003

[9] Chen, X., Wang, J., Shen, N., Luo, Y., Li, L., Liu, M. and Thomas, R.K. (2002) Gemini Surfactant/DNA Complex Monolayers at the Air-Water Interface: Effect of Surfactant Structure on the Assembly, Stability, and Topography of Monolayers. Langmuir, 18, 6222-6228. http://dx.doi.org/10.1021/la0256001

[10] Li, R., Chen, Q., Zhang, D., Liu, H. and Hu, Y. (2008) Mixed Monolayers of Gemini Surfactants and Stearic Acid at the Air/Water Interface. Journal of Colloid and Interface Science, 327, 162-168. http://dx.doi.org/10.1016/j.jcis.2008.07.038

[11] Acharya, D.P., Kunieda, H., Shiba, Y. and Aratani, K. (2004) Phase and Rheological Behavior of Novel Geini-Type Surfactant Systems. Journal of Physical Chemistry B, 108, 1790-1797. http://dx.doi.org/10.1021/jp036096b

[12] Rodriguez-Abreu, C., Rodriguez, E. and Solans, C. (2009) Monomeric and Dimeric Anionic Surfactants: A Comparative Study of Self-Aggregation and Mineralization. Journal of Colloid and Interface Science, 340, 254-260. http://dx.doi.org/10.1016/j.jcis.2009.08.043

[13] Yoshida, D., Yokoyama, T., Shimoaki, T., Tomita, T., Yoshida, T., Yamamoto, Y., Taga, K., Sumino, A., Dewa, T., Nango, M., Yamamoto, M. and Shervani, Z. (2013) Morphology Observation of Dipalmitoyl Phosphatidyl Choline (DPPC) Monolayer on Water Surface by Dropping Method. Biophysical Chemistry, 4, 115-121. http://dx.doi.org/10.4236/jbpc.2013.44016

[14] Mingotaud, A.F., Mingotaud, C. and Patterson, L.K. (1993) Handbook of Monolayers. Vol. 1 and 2, Academic Press Inc., California.

[15] Ohe, C., Ida, Y., Matsumoto, S., Sakaki, T., Goto, Y., Noi, M., Tsurumaru, T. and Itoh, K. (2004) Investigations of Polymyxin B-Phospholipid Interactions by Vibrational Sum Frequency Generation Spectroscopy. Journal of Physical Chemistry B, 108, 18081-18087. http://dx.doi.org/10.1021/jp0404347

[16] Frey, W., Schief Jr., W.R. and Vogel, V. (1996) Two-Dimensional Crystallization of Streptavidin Studied by Quantitative Brewster Angle Microscopy. Langmuir, 12, 1312-1320. http://dx.doi.org/10.1021/la9505374

[17] Minones Jr., J., Dynarowicz-Latka, P., Minones, J., Patino, J.M.R. and Iribarnegaray, E. (2003) Orientational Changes in Dipalmitoyl Phosphatidyl Glycerol Langmuir Monolayers. Journal of Colloid and Interface Science, 265, 380-385. http://dx.doi.org/10.1016/S0021-9797(03)00479-X

[18] Harkins, W.D. (1952) The Physical Chemistry of Surface Films. Reinhold, New York.

[19] Nakahara, H., Lee, S., Shoyama, Y. and Shibata, O. (2011) The Role of Palmitic Acid in Pulmonary Surfactant Systems by Langmuir Monolayer Study: Lipid-Peptide Interactions. Soft Matter, 7, 11351-11359. http://dx.doi.org/10.1039/c1sm06345f

[20] Nakahara, H. (2012) A Study on Novel Artificial Lung Surfactants Incorporated with Partially Fluorinated Amphiphiles. Yakugaku Zasshi, 132, 817-822. http://dx.doi.org/10.1248/yakushi.132.817 